\title{
EXPERIENCIAS RADIOFÓNICAS CON PERSONAS CON DISCAPACIDAD INTELECTUAL Y PARÁLISIS CEREBRAL EN ESPAÑA
}

\section{Radio experiences with people with intellectual disability and cerebral palsy in Spain}

Juan Antonio Cortés Fuentes

Dipalme Radio. Servicio Público de Radio de la Diputación de Almería

Beatriz Correyero RuIZ

Universidad Católica de Murcia. Facultad de Ciencias Sociales y de la Comunicación. Departamento de Periodismo. Campus de los Jerónimos, s/n. 30107 Guadalupe. Murcia

bcorreyero@ucam.edu

Recepción: 28 de enero de 2017

Aceptación definitiva: 27 de junio de 2018

Resumen: La radio es el medio de comunicación que mayor visibilidad puede dar a los colectivos en situación de exclusión social, y es también una herramienta que motiva la participación y contribuye al desarrollo integral de muchas personas, entre ellas, las que presentan alguna discapacidad intelectual o del desarrollo. En este trabajo se ha llevado a cabo una revisión y actualización de la base de datos de talleres de radio para personas con DI actualmente activos en España, a fecha de 2017, a través de los dos principales colectivos que representan a estas personas: la Confederación Española de Organizaciones a favor de las Personas con Discapacidad Intelectual (FEAPS) -hoy Plena inclusión-, plataforma que integra más de 800 asociaciones en todo el país, e incorporando la oferta de los talleres de la Confederación Española de Federaciones y Asociaciones de Atención a las personas con Parálisis Cerebral y Afines (Confederación ASPACE), que agrupa a unas 80 entidades en 17 comunidades autónomas. El resultado ofrece una foto fija de la estructura, funcionamiento y características de 53 proyectos radiofónicos en los que participan cerca de 500 personas con discapacidad intelectual.

Palabras clave: radio; discapacidad intelectual; taller de radio; educomunicación; empoderamiento; España. 
АвsтRACт: The radio is the communication media that can grant the greatest visibility to socially-excluded collectives, and it is also a tool that motivates participation. Thus, it contributes to the comprehensive development of many people, among which we find the collective of people with intellectual or developmental disability. In this research work, a review and update of the database of radio workshops for people with intellectual disability that are currently operational in Spain, until 2017, was conducted, through the main collectives that represent these individuals: the Spanish Confederation of Organizations in Support of Persons with Intellectual Disabilities (FEAPS), a platform that is comprised by more than 800 associations in the country, as well as the offerings by the workshops from the Spanish Confederation of Federations and Associations for the Care of Persons with Cerebral Palsy (ASPACE Confederation), which is comprised by 80 entities in 17 Autonomous Communities. The results offer a snapshot of the structure, functioning and characteristics of 53 radio projects, within which around 500 people with intellectual disability participate.

KEY WORDS: radio; intellectual disability; radio workshop; educommunication; empowerment; Spain.

\section{Introducción}

$\mathrm{D}$ esde la Declaración de los Derechos Humanos (ONU, 1948) hasta la Convención Internacional de los Derechos de las Personas con Discapacidad (ONU, 2006), las influencias del macrosistema en materia de derechos fundamentales han contribuido, como reflejan los principios de la Convención, a luchar contra la discriminación, a promover el respeto de la dignidad y por la diferencia, la igualdad de oportunidades, la accesibilidad y la participación e inclusión en la sociedad.

El presente estudio parte de la base de que estas dos últimas ideas: accesibilidad y participación, son claves para entender la eclosión del taller radiofónico como herramienta para dar una mayor visibilidad social y, a la par, empoderar a la persona con discapacidad. El taller es una expresión palpable del auge del asociacionismo de los colectivos con discapacidad.

La investigación que aquí se presenta es la continuación y revisión de un trabajo anterior (Cortés, 2016; Cortés y Correyero, 2017) que pretende dar a conocer el trabajo que realizan algunos colectivos dentro del movimiento de la Confederación Española de Organizaciones a favor de las Personas con Discapacidad Intelectual o del Desarrollo (FEAPS), hoy Plena inclusión, a través de la participación de estas personas en talleres de radio. En el primer estudio, realizado en 2014, se analizó por primera vez el funcionamiento de los talleres radiofónicos integrados por personas con discapacidad intelectual y se creó la primera base de datos sobre los mismos. En esta segunda investigación se han sumado a la muestra de estudio los talleres de la Confederación Española de Federaciones y Asociaciones de Atención a las personas con Parálisis Cerebral y Afines (ASPACE). El principal objetivo de este trabajo es conocer la evolución de los proyectos en los cuatro años transcurridos entre 2014 y 2017.

En el estudio inicial se detectaron 38 proyectos de comunicación radiofónica dependientes de FEAPS. Aunque algunos talleres han desaparecido: Prode de Cerca 
(Prode, Córdoba), Radio-teatro de Plena inclusión (Plena inclusión, Aragón), Somos uno más (Adisto, Comunidad Valenciana), Yo como tú (Plena inclusión, Madrid) y Mi voz importa (Plena inclusión, Baleares), observamos que en otros se han producido modificaciones tanto en el nombre del espacio radiofónico como en su organización. En el estudio de 2017 -con la colaboración de Dincat Cataluña y ASPACE-, el número de iniciativas reflejadas supera el medio centenar (53) y se ha recabado información sobre 20 talleres que no figuraban en la primera consulta.

\section{El papel social de la radio, herramienta de participación}

Dos son las corrientes teóricas que hemos estudiado para analizar las razones que llevan a sujetos con discapacidad intelectual (en adelante DI) o del desarrollo a participar en talleres de radio. Por un lado, nos interesaba conocer los beneficios personales que obtienen los participantes dentro del enfoque teórico de usos y gratificaciones cercano a los postulados funcionalistas (Levín y Denys, 2009). Por otro, nos planteábamos saber si la intervención en espacios radiofónicos puede empoderar al individuo mediante el desarrollo de habilidades comunicativas y sociales, la alfabetización mediática y la educomunicación. Consideramos que la alfabetización mediática es un derecho básico de la ciudadanía y un factor clave para la superación de barreras que dificultan la accesibilidad de los colectivos con discapacidad a los dispositivos tecnológicos (Varela, 2015; Jiménez-Lara, 2011) y a los entornos virtuales, así como "un elemento fundamental para lograr la inclusión social y digital de este sector de la población en la sociedad de la información y el conocimiento" (Bonilla, Valor y García-Ruiz, 2018).

Durante la realización de la investigación, y gracias a los testimonios de tutores y alumnos, se ha constatado que la elección de participar en un taller radiofónico es un acto libre, movido por el deseo de satisfacer unas necesidades básicas de los individuos que han configurado la muestra de este estudio. Como en su día apuntaron Katz, Gurevitch y Haas (1973), el uso de un medio como la radio puede satisfacer necesidades cognitivas (refuerzo de cultura), estético-afectivas, integradoras (estatus de personas informadas, mejora de la interacción social) y de evasión (entretenimiento, ocio). Siguiendo esta línea, nuestro objetivo pasaba por profundizar en las recompensas que depara a los participantes el proceso activo de colaboración en la radio.

Aunque la radio es un escenario de trabajo que puede deparar gratificaciones a los alumnos de los talleres, a la par puede ser útil para visibilizar sus realidades particulares y colectivas desde la creencia de que ayuda a construir un discurso propio. Esto explica que el medio radiofónico esté llamado a ser una eficaz herramienta para el empoderamiento social de las personas con DI y con parálisis cerebral.

\subsection{Educomunicación y alfabetización mediática: educar en y con los medios}

Los talleres de radio se erigen en recursos que muestran cómo funciona un medio de comunicación: sus procesos tecnológicos, de producción, redacción, locución o edición. 
Una vez alcanzado un conocimiento suficiente de los medios, el educando estará dotado de unas ciertas capacidades para apropiarse de ellos. Se parte, entonces, de la idea de que tan importante es el acceso a la radio como la usabilidad de la tecnología.

Además de esta visión mecanicista-materialista, los talleres contribuyen a educar en los medios y para los medios (educación mediática) favoreciendo la adquisición de códigos imprescindibles para discernir sus mensajes y entender sus contenidos. Este proceso, enmarcado dentro de la educación no formal, no pretende enseñar desde la perspectiva unilateral del educador, pues el educando es receptor y, también, emisor: aquí la comunicación es bidireccional. Nuestra investigación ha reflejado que la mayor parte de las personas con DI o del desarrollo y con parálisis cerebral incrementan su capacidad de discernimiento y, en consecuencia, tienen una idea más realista de la actualidad que les rodea.

Otro resultado de esta interacción es la adquisición de habilidades en materias como la comunicación verbal, lectoescritura, comprensión oral, escucha activa o creatividad.

En síntesis, los talleres de radio se engloban dentro de una educación basada en el proceso (Kaplún, 1998), cuyos fines son potenciar las capacidades intelectuales y la conciencia social de los educandos.

\subsection{Enfoque de usos y gratificaciones}

El estudio nos ha permitido corroborar que la actitud del alumno de los talleres de radio, en términos generales, es activa y participativa. El taller ofrece al usuario la posibilidad de edificar un discurso autónomo, aislado de los anhelos de persuasión de los mass-media: de los efectos. Cabe inferir en este punto que el educando ha elegido el medio con absoluta libertad por cumplir unas expectativas o necesidades concretas.

La exposición al medio radio, según el estudio, permite sentirse más feliz. Esta es la principal motivación, seguida de la necesidad de disfrutar de un tiempo de ocio, participar para relacionarse más e intervenir para que les conozcan mejor. Se advierte en las opiniones ofrecidas por los protagonistas de los talleres de radio que la mejora del estado de ánimo, el bienestar personal, es una motivación transversal para una mayoría de usuarios. Se trata de necesidades abstractas que encuentran su raíz en el plano psicológico o afectivo. Se puede argumentar que las necesidades de participación entroncan con las cinco dimensiones de la discapacidad intelectual propuestas en la última clasificación de la American Association on Intellectual and Developmental Disabilities (AAIDD, 2011):

- La dimensión de "Habilidades Intelectuales" se refleja en el deseo de razonar sobre el entorno que les rodea y el sentido crítico de la realidad.

- La dimensión de "Conducta Adaptativa" se observa en la potenciación de habilidades comunicativas y sociales.

- La dimensión de "Salud" encuentra explicación en el bienestar emocional que puede proporcionar el acceso a la radio y su uso y disfrute. Sobre este tema es muy interesante el estudio llevado a cabo por Sande y Leal (2015). 
- La dimensión de "Participación" es visible en la interacción con amigos, compañeros de ocio, audiencia o comunidad educativa que establece el individuo con DI y con parálisis cerebral.

- La dimensión de "Contexto" está relacionada con las condiciones en las que disfrutan su experiencia los usuarios de los talleres: ambiente físico o social o características personales.

En resumen, las necesidades se acercan a la naturaleza del modelo socioecológico de la discapacidad. Un modelo en el que las limitaciones no son una realidad invariable y pueden cambiar sustancialmente merced a la interacción entre la persona y su entorno (Schalock, Verdugo y Lee, 2016; Schalock, 2009). En ese entorno situamos como apoyo el taller de radio.

\section{Método}

La investigación sobre la participación de personas con discapacidad intelectual o del desarrollo en talleres de radio encuentra su raíz en el movimiento asociativo FEAPS -hoy Plena inclusión-, una plataforma que integra a más de 800 asociaciones y entidades de toda España, de gran prestigio institucional. Pero también se ha contado con la colaboración de la Confederación Española de Federaciones y Asociaciones de Atención a las personas con Parálisis Cerebral y Afines (Confederación ASPACE), un organismo que agrupa a unas 80 entidades también repartidas por todo el territorio nacional.

En esta investigación se han triangulado técnicas cuantitativas y cualitativas de recogida de datos como parte de una metodología descriptiva. La muestra está compuesta por tres grupos de individuos:

El primero lo conforman los directores de comunicación de las Federaciones regionales y de las Delegaciones provinciales de FEAPS y ASPACE. Con ellos se contactó telefónicamente para rastrear y localizar los talleres de radio dentro de cada asociación. El periodo de recogida de datos se ha realizado en dos fechas distantes en el tiempo: de enero a julio de 2014 (Cortés, 2016) y entre febrero y marzo de 2017. En la primera fase respondieron 38 colectivos (Cataluña fue la única comunidad autónoma que no respondió a la demanda de información). En la segunda fase respondieron a la demanda de información 20 organizaciones que contaban con un taller de radio. En la Tabla 2 se recoge el listado de los talleres participantes en la investigación. Para realizar este artículo hemos cruzado los principales datos de la investigación inicial confrontándolos y enriqueciéndolos con las nuevas aportaciones localizadas en el último estudio

Aunque es probable que muchos proyectos no hayan sido identificados -la muestra final (53 talleres) recoge aquellos que contestaron el cuestionario-, estimamos que es un reflejo fiel y representativo para extrapolar los resultados y poder realizar una foto fija del estado de este tipo de iniciativas radiofónicas en nuestro país.

El segundo grupo de individuos que han participado en el estudio está compuesto por los tutores y responsables de los talleres. A ellos se les envió un cuestionario 
semiestructurado (Cortés, 2016) que trataba de recabar datos sobre la estructura, composición y organización de un taller radiofónico, con objeto de crear una base de datos de talleres de radio en nuestro país. Las preguntas versaban sobre las siguientes variables.

- Tipo de emisión

- Periodicidad de las emisiones

- Tipología de emisora

- Tipología de programas

- Géneros radiofónicos que más se trabajan

- Número de participantes

- Perfil de los alumnos

- Requisitos para participar

- Tareas y ocupaciones de los DI en el taller

- Percepción de los tutores sobre la utilidad de los talleres de radio.

El tercer grupo muestral lo conforman los usuarios con DI o del desarrollo. Ellos también tuvieron que responder a un cuestionario estructurado, validado previamente por un grupo de ocho tutores seleccionados según criterios de experiencia, competencia y disposición a colaborar. En esta ocasión, el principal objetivo de la investigación era conocer la influencia de los talleres de radio en las personas con DI o del desarrollo mediante el análisis de la utilización que hacen de estos espacios y las gratificaciones que obtienen. El cuestionario pretendía extraer datos sobre las siguientes variables:

- Rasgos sociodemográficos de los participantes en los talleres de radio;

- Características de los tutores de los proyectos; y

- Percepción de los usuarios sobre su participación en los talleres, gratificaciones y grado de satisfacción.

En total, el número de participantes en esta segunda encuesta fue de 175, una tercera parte del total de alumnos de los talleres reflejados en la base de datos (unos 600).

\section{Resultados}

\subsection{Funcionamiento de un taller de radio}

En 2017, el listado de talleres ha sufrido variaciones notables. Se mantienen la mayor parte de los proyectos detectados en 2014 y han aparecido nuevos talleres radiofónicos. En la Tabla 1 figuran 53.

En el estudio de 2014, 14 comunidades autónomas disponían de talleres de radio con participación de personas con DI o del desarrollo: Andalucía, Aragón, Asturias, Baleares, Cantabria, Castilla-La Mancha, Castilla y León, Comunidad Valenciana, Extremadura, Galicia, La Rioja, Madrid, Melilla y Murcia.

En el primer estudio de 2014, la Comunidad de Madrid era la región con mayor número de iniciativas (el 31\%). Andalucía y Castilla y León ocupaban el segundo y tercer puesto respectivamente $(13 \%)$. 


\begin{tabular}{|c|c|c|c|}
\hline \multicolumn{2}{|c|}{ TAlleres 2014} & \multicolumn{2}{|c|}{ Talleres 2017} \\
\hline Andalucía & 5 & Andalucía & 9 \\
\hline Aragón & 3 & Aragón & 3 \\
\hline Asturias & 1 & Asturias & 1 \\
\hline Baleares & 1 & Canarias & 1 \\
\hline Cantabria & 2 & Cantabria & 2 \\
\hline Castilla-La Mancha & 2 & Castilla-La Mancha & 3 \\
\hline Castilla y León & 5 & Castilla y León & 6 \\
\hline- & - & Cataluña & 4 \\
\hline Comunidad Valenciana & 1 & Comunidad Valenciana & 1 \\
\hline Extremadura & 2 & Extremadura & 2 \\
\hline Galicia & 1 & Galicia & 2 \\
\hline La Rioja & 1 & La Rioja & 1 \\
\hline Madrid & 12 & Madrid & 14 \\
\hline Melilla & 1 & Melilla & 1 \\
\hline Murcia & 1 & Murcia & 2 \\
\hline- & - & Navarra & 1 \\
\hline Total & 38 & Total & 53 \\
\hline
\end{tabular}

Fuente: Elaboración propia.

En el listado de 2017, Madrid había incrementado el número de proyectos (14) y en Andalucía crecían más de un 40\% (9). También hubo aumento de espacios en Castilla-La Mancha y Castilla y León, Galicia y Murcia. Navarra (1) y Canarias (1) entraron en la lista y en Cataluña se detectaron 4.

\begin{tabular}{|l|l|l|l|l|}
\hline \multicolumn{5}{|c|}{ TABLA 2. Base de datos de talleres de radio por regiones (2017) } \\
\hline \multirow{5}{*}{ ANDALUCÍA } & Almería & A TODA VELA & A TODA RADIO & RNE/DIPALME RADIO \\
\cline { 2 - 5 } & Almería & ASPAPROS & COMPARTIENDO SUEÑOS & DIPALME RADIO, IVOOX \\
\cline { 2 - 5 } & Córdoba & DISGENIL & HACIENDO AMIGOS & RADIO SINTONÍA, IVOOX \\
\cline { 2 - 5 } & Granada & ASPROGRADES & MAGIA EN LA RADIO & IVOOX \\
\cline { 2 - 5 } & $\begin{array}{l}\text { ASOC. PRO- } \\
\text { DISCAPACITADOS } \\
\text { JABALCÓN }\end{array}$ & INTEGRADOS EN LA ONDA & ONDA CERO \\
\cline { 2 - 5 } & Granada & ASOC. VALE & VALE EN EL AIRE & RADIO DÚRCAL, IVOOX \\
\cline { 2 - 5 } & Málaga & AMAPPACE & ENREDA2 RADIO & $\begin{array}{l}\text { WEB www.enred22mx; } \\
\text { CANAL PODCAST DE AMAPPACE }\end{array}$ \\
\cline { 2 - 5 } & Sevilla & ADEFISAL & TE LO CONTAMOS & SOLÚCAR RADIO, IVOOX \\
\cline { 2 - 5 } & Sevilla & ASASY ASPACE & RUEDAS Y PIERNAS & IVOOX \\
\hline
\end{tabular}

Fuente: Elaboración propia. 
EXPERIENCIAS RADIOFÓNICAS CON PERSONAS CON DISCAPACIDAD INTELECTUAL

Y PARÁLISIS CEREBRAL EN ESPAÑA

JUAN ANTONIO CORTÉS FUENTES Y BEATRIZ CORREYERO RUIZ

\begin{tabular}{|c|c|c|c|c|}
\hline \multirow{3}{*}{ ARAGÓN } & Huesca & $\begin{array}{l}\text { CADIS, ASPACE DOWN, } \\
\text { CRUZ BLANCA, ATADES } \\
\end{array}$ & BANDIDOS DE LA HOYA & $\begin{array}{l}\text { RADIO HUESCA, FACEBOOK CADIS } \\
\text { HUESCA }\end{array}$ \\
\hline & Zaragoza & AMIBIL & LA VOZ DE AMIBIL & CADENA SER, FACEBOOK AMIBIL \\
\hline & Zaragoza & ASPACE & $\begin{array}{l}\text { RADIO ASPACE ZARAGOZA } \\
\text { 'PRÉSTANOS TUS OÍDOS' } \\
\end{array}$ & $\begin{array}{l}\text { BLOG: prestanostusoidos.blogspot.com, } \\
\text { IVOOX }\end{array}$ \\
\hline ASTURIAS & Asturias & ASOC. REY PELAYO & EL MICRÓFONO & LA RADIADORA, IVOOX \\
\hline CANARIAS & Las Palmas & $\begin{array}{l}\text { ASOC. SÍNDROME DE } \\
\text { DOWN } \\
\end{array}$ & $\begin{array}{l}\text { 'MÁS DE 21' (RADIO ROSCAS } \\
\text { Y COTUFAS) }\end{array}$ & CANARIAS RADIO, IVOOX \\
\hline \multirow[b]{2}{*}{ CANTABRIA } & Cantabria & AMPROS & RADIO ALTERNATIVA & INTERNA \\
\hline & Cantabria & $\begin{array}{l}\text { C. E. ESPECIAL 'DR } \\
\text { FERNANDO ARCE' }\end{array}$ & $\begin{array}{l}\text { RADIO MENUDA, MENUDA } \\
\text { RADIO }\end{array}$ & INTERNA \\
\hline \multirow{3}{*}{$\begin{array}{l}\text { CASTILLA } \\
\text {-LA MANCHA }\end{array}$} & Albacete & ASPRONA & CON BUENA ONDA & NOVA ONDA, FACEBOOK NOVA ONDA \\
\hline & \begin{tabular}{|l} 
Ciudad \\
Real
\end{tabular} & LABORVALÍA & SER CAPACES & CADENA SER, IVOOX \\
\hline & Toledo & DOWNTOLEDO & CAPACES EN LA ONDA & ONDA CERO, IVOOX \\
\hline \multirow{6}{*}{$\begin{array}{l}\text { CASTILLA } \\
\text { Y LEÓN }\end{array}$} & Palencia & $\begin{array}{l}\text { ASPANIS, FUNDACIÓN } \\
\text { PERSONAS }\end{array}$ & NUEST'RA VOZ IMPORTA & $\begin{array}{l}\text { RADIO COLORES, FACEBOOK RADIO } \\
\text { COLORES }\end{array}$ \\
\hline & Palencia & \begin{tabular}{|l} 
FUNDACIÓN SAN \\
CEBRIÁN \\
\end{tabular} & $\begin{array}{l}\text { EL CIMBALILLO DE LA } \\
\text { FUNDACIÓN } \\
\end{array}$ & ONDA JUDÍA, IVOOX ONDA JUDÍA \\
\hline & Palencia & $\begin{array}{l}\text { FUNDACIÓN SAN } \\
\text { CEBRIÁN }\end{array}$ & $\begin{array}{l}\text { EMOCIÓNATE RADIO } \\
\text { (ANTES: VOCES QUE } \\
\text { CUENTAN) }\end{array}$ & $\begin{array}{l}\text { IVOOX/YOUTUBE (EMISIONES } \\
\text { COMPLETAS)/INTERNA }\end{array}$ \\
\hline & Salamanca & \begin{tabular}{|l} 
ASPRODES-FEAPS \\
SALAMANCA \\
\end{tabular} & $\begin{array}{l}\text { RADIO PICADILLO (ANTES: } \\
\text { CONCIERTO SENTIDO) }\end{array}$ & $\begin{array}{l}\text { RADIO OASIS, IVOOX, FACEBOOK } \\
\text { RADIO OASIS } \\
\end{array}$ \\
\hline & Salamanca & ASPRODES & ESPACIO ASPRODES & RADIO VITIGUDINO \\
\hline & Valladolid & $\begin{array}{l}\text { FUNDACIÓN PERSONAS, } \\
\text { ASPRONA } \\
\end{array}$ & TALLER RADIO EL PINO & RADIO EL PINO (EMISORA EDUCATIVA) \\
\hline \multirow{4}{*}{ CATALUÑA } & Girona & FUNDACIÓ ESTANY & 7 DE RÀDIO & RADIO BANYOLES \\
\hline & Barcelona & $\begin{array}{l}\text { DINCAT (ENTIDAD } \\
\text { COLABORADORA) } \\
\end{array}$ & CLUB DELS BOLATS & RADIO SAN CUGAT, IVOOX \\
\hline & Barcelona & FUNDACIÓ EL RUSC & DIVERSONES & RADIO TORDERA, IVOOX \\
\hline & Barcelona & ENCERT & ENCERT A LA RADIO & RADIO BOCA, IVOOX \\
\hline $\begin{array}{l}\text { COMUNIDAD } \\
\text { VALENCIANA }\end{array}$ & Valencia & $\begin{array}{l}\text { CENTRO OCUPACIONAL } \\
\text { MAIGMO } \\
\end{array}$ & $\begin{array}{l}\text { SECCIÓN MAIGMO } \\
\text { EN 'SAN VICENTE PLAZA' }\end{array}$ & RADIO SAN VICENTE, IVOOX \\
\hline \multirow{2}{*}{ EXTREMADURA } & Badajoz & APROSUBA 1 & LA VENTANA ABIERTA & RADIO EMISUR \\
\hline & Cáceres & PLÁCEAT & LA TERTULIA DE PLÁCEAT & CADENA SER \\
\hline \multirow{2}{*}{ GALICIA } & A Coruña & ASPACE & RADIO DE LOS GATOS & IVOOX, YOUTUBE \\
\hline & Vigo & APAMP-ASPACE & RADIO APAMPDA & YOUTUBE, WEB DE APAMP \\
\hline LA RIOJA & La Rioja & IGUAL A TI & ÉSTA ES NUESTRA VOZ & $\begin{array}{l}\text { RADIO AUTOL, WEB: } \\
\text { http:///estaesnuestravoz.org/programas-de-radio }\end{array}$ \\
\hline
\end{tabular}

Fuente: Elaboración propia.

(C) Ediciones Universidad de Salamanca / CC BY-NC-ND

Siglo Cero, vol. 50 (1), n. ${ }^{\circ}$ 269, 2019, enero-marzo, pp. 75-95 
EXPERIENCIAS RADIOFÓNICAS CON PERSONAS CON DISCAPACIDAD INTELECTUAL

Y PARÁLISIS CEREBRAL EN ESPAÑA

JUAN ANTONIO CORTÉS FUENTES Y BEATRIZ CORREYERO RUIZ

\begin{tabular}{|c|c|c|c|c|}
\hline \multicolumn{5}{|c|}{ TABLA 2. Base de datos de talleres de radio por regiones (2017) (cont.) } \\
\hline \multirow{14}{*}{ MADRID } & Madrid & ADISLI & ADISLEANDO & RADIO ENLACE, IVOOX \\
\hline & Madrid & APMIB-ENVERA & RADIO TERRÍCOLA & WEB: radioterricola.grupoenvera.org \\
\hline & Madrid & APAMA & TAMBIÉN CONTAMOS & RADIO UTOPÍA, IVOOX \\
\hline & Madrid & APROCOR & VOCES DE APROCOR & RADIO ENLACE, IVOOX \\
\hline & Madrid & FUNDACIÓN BOBATH & RADIORED & RADIO VALLEKAS, RADIORED \\
\hline & Madrid & $\begin{array}{l}\text { FUNDACIÓN INSTITUTOO } \\
\text { SANJOSÉ }\end{array}$ & VOCES PARA EL CORAZÓN & FACEBOOK/YOUTUBE/INTERNA \\
\hline & Madrid & \begin{tabular}{|l} 
FUNDACIÓN GIL \\
GAYARRE \\
\end{tabular} & $\begin{array}{l}\text { RADIO ALEGRÍA, EDICIÓN } \\
\text { POZUELO }\end{array}$ & RADIO ENLACE, IVOOX \\
\hline & Madrid & $\begin{array}{l}\text { FUNDACIÓN GIL } \\
\text { GAYARRE }\end{array}$ & $\begin{array}{l}\text { RADIO ALEGRÍA, EDICIÓN } \\
\text { LA GRANJA }\end{array}$ & RADIO ENLACE, IVOOX \\
\hline & Madrid & FUNDACIÓN JUAN XXIII & \begin{tabular}{|l|} 
RADIO RONCALLI(ANTES \\
RADIO O LO QUE SEA) \\
\end{tabular} & RNE, IVOOX \\
\hline & Madrid & $\begin{array}{l}\text { FUNDACIÓN SÍNDROME } \\
\text { DE DOWN }\end{array}$ & GENTE EXTRAORDINARIA & GESTIONA RADIO, IVOOX \\
\hline & Madrid & $\begin{array}{l}\text { FUNDACIÓN SÍNDROME } \\
\text { DE DOWN } \\
\end{array}$ & CAFÉ CON LUPA & RADIO ALMENARA, IVOOX \\
\hline & Madrid & FUNDACIÓN TRÉBOL & EL VALOR DE OTRAS VOCES & RADIO MARÍA, IVOOX \\
\hline & Madrid & GRUPO AMÁS & EL OÍDO DEL SALTAMONTES & ECOLEGANÉS, WEB: audio.urcm.net \\
\hline & Madrid & CIRVITE & LA TILDE DE CIRVITE & RADIO ENLACE, IVOOX \\
\hline MELILLA & Melilla & ASPANIES-FEAPS MELILLA & CAPACES & ONDA CERO \\
\hline \multirow{2}{*}{ MURCIA } & Murcia & ASSIDO & LA RADIO DE ASSIDO & IVOOX \\
\hline & Murcia & ASTRAPACE & COLGADOS EN LA ONDA & ONDA REGIONAL, IVOOX \\
\hline NAVARRA & Navarra & ASPACE & LOS GRANUJAS DEL NORTE & WEB ASPACE NAVARRA, YOUTUBE \\
\hline
\end{tabular}

Fuente: Elaboración propia.

\subsubsection{Media de participantes}

Para conocer el número aproximado de participantes por taller decidimos incorporar nueve horquillas con oscilaciones de cinco unidades, según la información previa facilitada por los tutores: 1-5, 6-10, 11-15, 16-20, 21-25, 26-30, 31-35, 46-50 y 151-155. La participación media fluctuaba entre 6 y 15 colaboradores.

Radio Terrícola, primera radio hecha por personas con DI o del desarrollo, lideraba el listado con una participación media de unos 150 integrantes. El dato responde a la lógica toda vez que Radio Terrícola no es un programa o una sección al uso, sino una radio online que emite secuencias de pódcast.

Otros proyectos numerosos eran Bandidos de la Hoya, La Radio de Assido, Radio Picadillo (antes Concierto Sentido), Ruedas y Piernas, Radio Menuda, Menuda Radio o La Radio de los Gatos. 


\subsubsection{Tipo de emisión: FM, Internet, Radio interna}

En el estudio de 2014, el 71\% de los talleres emitían en FM y el 89\% a través de Internet vía online o mediante pódcast. De los proyectos que se escuchaban en Internet-solo cuatro carecían de proyección digital: A toda radio, Espacio Asprodes, Radio Menuda, Menuda Radio y Radio Alternativa-, el 85\% disponían regularmente de servicio de pódcast.

En 2017 comprobamos que, de las 53 iniciativas detectadas, el 68\% (36 talleres) emitían en emisoras de FM -porcentaje parecido al registrado en 2014-, mientras que solo el 32\% (17) quedaban fuera de las ondas.

$\mathrm{Si}$ analizamos la incursión en Internet, el 11\% de los proyectos estaba fuera del ciberespacio, de modo que nueve de cada diez talleres tenían presencia en la Red-con emisiones online en las páginas de las emisoras o mediante pódcast en Ivoox, Facebook, Youtube o páginas web-.

De estos datos se infiere que la radio tradicional continúa siendo un cauce natural para los colectivos de personas con DI o del desarrollo y parálisis cerebral. Numerosas asociaciones y entidades han llegado a acuerdos con emisoras locales o regionales de FM para realizar programas o secciones.

Además de las emisiones en FM y en Internet, en la investigación detectamos la presencia de talleres de radio internos que presentan dos modalidades:

- En el primer grupo están aquellos que emiten en el interior de un centro usando el canal de megafonía (Radio menuda, Menuda Radio y Radio Alternativa) para satisfacer unas necesidades concretas de la comunidad donde viven, trabajan o estudian los usuarios. Las emisiones se hacen con una cierta regularidad y el proceso de producción es radiofónico.

- En un segundo grupo encontramos talleres que son grabados regularmente y se proyectan exclusivamente en plataformas de podcasting y/o en redes sociales, y proyectos que funcionan con regularidad -producen y graban habitualmente-, aunque sus emisiones en FM son muy esporádicas.

\subsubsection{Tipología de las emisoras colaboradoras: privadas, públicas, comunitarias}

La genealogía de las emisoras colaboradoras ofrece tres modalidades: emisoras privadas, emisoras públicas y emisoras comunitarias o culturales. En 2014, un 40\% de los talleres se realizaban en estaciones comunitarias. Las emisoras públicas representaban un $30 \%$. En este grupo, cerca del $80 \%$ eran municipales, dependientes de ayuntamientos. Y otro $30 \%$ de los talleres se proyectaban en emisoras privadas.

En el estudio de 2017, se contabilizaron catorce talleres en radios culturales o comunitarias - dos más que en 2014-, 16 talleres se emitían en radios públicas -nueve, en 2014y nueve en radios de índole privada -nueve también se registraron en el primer estudio-.

En la órbita de las emisoras comunitarias, Radio Enlace lideraba el listado del segundo estudio con cinco proyectos - la emisora alberga en la actualidad en torno a una decena de iniciativas radiofónicas de distintos grupos relacionados con la discapacidad-. 
Dentro de las emisoras públicas, la radio municipal es una de las opciones más accesibles para los colectivos de personas con discapacidad, pese a que la crisis económico-financiera de los últimos años ha provocado el cierre de emisoras y, sobre todo, recortes presupuestarios que han mermado sus programaciones. Esto es debido a la autonomía en la gestión horaria, a su espíritu de servicio público y a la cercanía con el asociacionismo.

\subsubsection{Son nuevos tiempos: proyectos independientes}

El mayor crecimiento en estos tres años se ha vivido dentro del grupo de las iniciativas independientes de emisoras de radio públicas, privadas y comunitarias-culturales. En el estudio de 2017 descubrimos 14 proyectos, de los que solo dos quedaban fuera de la órbita de Internet.

Hablamos de asociaciones y entidades que graban pódcast aprovechando las potencialidades de plataformas como Ivoox o a través de páginas web, Facebook o Youtube. Estos talleres se difunden en las redes sociales o en las direcciones web de los colectivos que representan a los alumnos.

\begin{tabular}{|l|l|l|}
\hline \multicolumn{3}{|c|}{ TABLA 3. Talleres independientes de emisoras de radio (2017) } \\
\hline Taller & Colectivo & Canal \\
\hline Magia en la radio & (ASPROGRADES, Granada) & IVOOX \\
\hline Enreda2 Radio & (AMAPPACE, Málaga) & $\begin{array}{l}\text { WEB www.enreda2.mx; CANAL } \\
\text { PODCAST DE AMAPPACE }\end{array}$ \\
\hline Ruedas y piernas & (ASAS Y ASPACE, Sevilla) & IVOOX \\
\hline Radio ASPACE Préstanos tus oídos & (ASPACE, Zaragoza) & $\begin{array}{l}\text { BLOG: prestanostusoidos.blogspot. } \\
\text { com, IVOOX }\end{array}$ \\
\hline Radio Alternativa & (AMPROS, Cantabria) & MEGAFONÍA INTERNA \\
\hline Radio Menuda, Menuda Radio & $\begin{array}{l}\text { (C. E. ESPECIAL 'DR. FERNANDO } \\
\text { ARCE', Cantabria) }\end{array}$ & $\begin{array}{l}\text { MEGAFONÍA INTERNA EN } \\
\text { TALLER DE RADIO ESCOLAR }\end{array}$ \\
\hline $\begin{array}{l}\text { Emociónate Radio (en 2014: Voces que } \\
\text { cuentan) }\end{array}$ & $\begin{array}{l}\text { (FUNDACIÓN SAN CEBRIÁN, } \\
\text { Palencia) }\end{array}$ & $\begin{array}{l}\text { IVOOX/YOUTUBE (EMISIONES } \\
\text { COMPLETAS)/INTERNA }\end{array}$ \\
\hline Taller Radio El Pino & $\begin{array}{l}\text { (FUNDACIÓN PERSONAS, } \\
\text { ASPRONA, Valladolid) }\end{array}$ & TALLER DE RADIO ESCOLAR \\
\hline Radio de los Gatos & (ASPACE, A Coruña) & IVOOX, YOUTUBE \\
\hline Radio APAMPDA & (APAM, ASPACE, Vigo) & YOUTUBE, WEB DE APAMP \\
\hline Radio Terrícola & (ENVERA, Madrid) & WEB: radioterricola.grupoenvera.org \\
\hline Voces para el corazón & $\begin{array}{l}\text { (FUNDACIÓN INSTITUTO SAN } \\
\text { JOSÉ, Madrid) }\end{array}$ & $\begin{array}{l}\text { FACEBOOK/YOUTUBE/ } \\
\text { INTERNA }\end{array}$ \\
\hline Radio Assido & (ASSIDO, Murcia) & IVOOX \\
\hline Los granujas del Norte & (ASPACE, Navarra) & $\begin{array}{l}\text { YOUTUBE, WEB ASPACE } \\
\text { NAVARRA }\end{array}$ \\
\hline
\end{tabular}

Fuente: Elaboración propia. 
Radio Terrícola es un caso muy particular. Inaugurada el 26 de junio de 2013 en el centro de terapia ocupacional de Colmenar Viejo (Madrid) de la antigua Asociación de Empleados de Iberia (APMIB) -hoy Grupo Envera-, se trata de la primera radio online realizada por personas con DI. Aunque ha dejado de emitir temporalmente vía streaming, sus principales programas se pueden escuchar vía pódcast a través de la página web: http://radioterricola.grupoenvera.org.

Las entrevistas a personajes relevantes son una de las secciones destacadas. En 2017, los usuarios tuvieron la oportunidad de hablar con Kiko Narváez, Georgina León o Soledad Becerril.

En la categoría de Actualidad, Radio Terrícola emite los espacios La Pecera, Crónicas terrícolas y Crónicas. En materia de Ocio, ofrece espacios como El Finde, Kikirikí con Killo, Érase una vez (cuenta-cuentos) y el programa deportivo El pichichi.

La radio propone programas especiales (San Valentín, Navidad, Día Internacional contra la Violencia de género o Día Internacional de las Personas con Discapacidad). Otros espacios interesantes son El bargueño, Desde el okupa con amor o Tutorías en las ondas.

En la Web publican también informaciones actualizadas con un resumen de los protagonistas de los principales espacios de la radio, acompañadas de imágenes.

Entre los fines de este medio, Envera destaca la consecución de aprendizajes en áreas como las relaciones sociales, la expresión oral y escrita, el trabajo en equipo, la memoria auditiva y la capacidad de escucha. Es, en definitiva, un canal de expresión en el que personas con capacidades diferentes acercan a la sociedad sus propios discursos y, en paralelo, mejoran sus competencias comunicativas y sociales.

\subsubsection{Programa o sección de un programa}

Si analizamos los dos trabajos de campo $(2014,2017)$, la mayor parte de los talleres guardaban las características de un programa de radio, mientras que la colaboración en secciones de programas representaba una sexta parte del total. En el trabajo realizado tan solo hace unos meses, el 16,9\% (9) eran secciones. El resto, un $83 \%$, se pueden considerar programas (44).

Los programas eran espacios dotados de independencia dentro de una programación de una emisora de radio o alojados en plataformas de pódcast. En ambos casos, tenían los rasgos típicos de un programa: emancipación del resto de unidades de la programación, alternancia de géneros, secciones variadas y una duración concreta -habitualmente, entre media hora y una hora-.

Las emisoras que acogían estos programas eran especialmente las radios comunitarias y municipales. La explicación está en que estas estaciones cuentan con más autonomía y horario disponible para diseñar parrillas más diversas.

Dentro de la categoría de programas-pódcast encontramos espacios almacenados en yacimientos de audios como Ivoox y/o o en redes sociales.

En contraste, la mayoría de los talleres que colaboraban en secciones de programas se alojaban en las desconexiones territoriales de las delegaciones de emisoras de ámbito nacional y, excepcionalmente, en emisoras regionales (Canarias Radio, Onda Regional de Murcia). 


\subsubsection{Periodicidad de las emisiones}

En la investigación se descubrió que la mayor parte de los espacios tenían una periodicidad semanal-quincenal o mensual -alrededor del $90 \%-$, mientras que solo una minoría emitía varios días a la semana o sin una periodicidad fija.

La ocupación horaria -mañana, mediodía, tarde- no ofrece información relevante ya que no se aprecia una franja claramente dominante. No obstante, con la incorporación de los pódcast han aumentado los espacios que carecen de un horario fijo.

Caso especial es el de Radio Terrícola, que emitía programación durante todo el día en el momento de la investigación de 2014, aunque desde hace unos meses ha dejado sus transmisiones en streaming.

Con respecto al tiempo de emisión, la duración media es entre media hora y una hora. Las secciones tienen una duración menor: entre quince minutos y media hora.

\subsubsection{Perfil de los alumnos}

En la distribución de frecuencias de la encuesta realizada a los educandos de los talleres, cursada en 2015-2016 para la tesis doctoral, se observaba que la participación masculina era 17 puntos superior a la femenina. La media de edad de los usuarios era de 40,2 años. Este dato alteró nuestra idea inicial de que los usuarios eran más jóvenes y vino a confirmar que los intervinientes en los talleres suelen ser personas adultas que eligen en función de una necesidad. En las franjas de edad de 50 a 70 años constatamos la presencia de más de una treintena de participantes, aunque el mayor porcentaje de participación se da en el intervalo de los 30 a los 40 años.

Si examinamos el grado de autonomía, un 66,5\% vivían con la familia, cerca de un $30 \%$ en un centro y apenas un $4 \%$ admitían vivir solos. Al cruzar las variables 'Grado de Autonomía' y 'Edad' nos percatamos de que, hasta los 50 años, la mayoría de los encuestados residían con familiares y a partir de los 50 los usuarios que viven en un centro superan en cantidad a aquellos que residen en casa.

En torno al $56 \%$ de los alumnos carecían de estudios, aunque se aprecia que las generaciones más jóvenes han tenido más oportunidades de acceso a la educación. Y es que, a partir de los 50 años, el 78,7\% reconocieron no contar con formación académica.

Al estudiar la situación laboral nos cercioramos de que casi las dos terceras partes $(63 \%)$ de quienes contestaron al cuestionario estaban en el paro y cerca de una quinta parte $(21,2 \%)$ no estaban en condiciones para trabajar.

\subsubsection{Perfil de los tutores}

En el área formativa, los tutores que coordinan a los alumnos de talleres de radio pueden agruparse en dos grupos: aquellos que han realizado estudios en ámbitos como Psicología, Pedagogía, Magisterio o Trabajo Social, y aquellos otros que tienen formación en materia audiovisual. 
Además de la coordinación de la actividad radiofónica, los tutores ejercen distintas labores dentro de los colectivos de Plena inclusión y ASPACE: coordinadores y/o monitores de actividades de ocio, responsables de programas de apoyo escolar, cuidadores, profesores de cualificación profesional, psicólogos de la asociación/entidad o periodistas del área de Comunicación.

Las tareas que desempeña el tutor dentro del taller de radio son dos: coordinar los grupos y servir de apoyo. El 80\% aseguraron que su ocupación principal son las labores de organización, mientras que el resto consideraban que su misión más importante era la de orientar y apoyar a los usuarios en un segundo plano.

\subsubsection{Requisitos para participar en los talleres}

Por lo general, los participantes son usuarios de los colectivos. La mayor parte de las asociaciones no realizan filtros de una gran exigencia a los interesados. Los requisitos son mínimos y, por lo que se desprende de las respuestas de los tutores, el principal requerimiento es la avidez del usuario por ser copartícipe del taller de radio.

En segundo lugar, se aconseja un nivel mínimo de lectoescritura y una expresión oral comprensible, aunque las personas a las que les cuesta escribir y que no hablan con claridad pueden realizar otras tareas dentro de las áreas técnica y de producción.

\subsubsection{Secciones y principales géneros de los talleres}

Generalmente, los espacios son magacines en los que la información especializada (discapacidad), la información general, la música, los deportes y el ocio ofrecen contenidos corales.

La actualidad del colectivo y la información sobre discapacidad es la categoría con mayor prevalencia dentro de los talleres. Tanto los tutores, en el primer cuestionario, como los usuarios, en el segundo, reconocieron que dan prioridad a estas temáticas. De hecho, un tercio de los alumnos contestaron que la principal sección es trasladar noticias relacionadas con el trabajo de la asociación y vinculadas a la discapacidad.

La transmisión de informaciones de carácter general del entorno -ciudad, pueblo- y del ámbito político y social figuran como opciones preferentes dentro de los guiones.

La música es una realidad transversal en los espacios. Las voces de los locutores se alternan con canciones dedicadas, música para separar secciones o noticias musicales. La música puede estar en primer plano o en plano de fondo, ambientando los temas que se abordan en los programas.

Los deportes, sobre todo los más locales, tienen cabida periódica en los talleres de radio como secciones diferenciadas o como base de debate para tertulias.

Sobre los géneros, la noticia está presente en la mayoría de guiones, las entrevistas a personajes relevantes o a ciudadanos anónimos son una herramienta muy habitual en los programas y también son frecuentes las tertulias en las que los usuarios trabajan en equipo. 


\subsubsection{Tareas y ocupaciones dentro del taller}

La mayoría de talleres de radio realizan un completo proceso creativo. Los usuarios participan en la preproducción, producción y, en ciertas ocasiones, la postproducción -es el caso de aquellos espacios grabados-. En la etapa de producción, los educandos del taller desarrollan trabajos como la puesta en común de ideas, el diseño de un guion, la búsqueda de recursos para investigar o la asignación de temas. Hay proyectos en los que, con grupos estables, el reparto de responsabilidades está asumido de antemano, pero en otros colectivos la participación cambia frecuentemente, por lo que, de forma periódica, se produce una adjudicación de ocupaciones. Estos asuntos suelen ser tratados en reuniones previas o de coordinación.

La labor de redacción recae en personas con un nivel de lectoescritura suficiente -el $88,8 \%$ de los usuarios dijeron en la encuesta que controlan la escritura y un $89,5 \%$ manifestó dominar la lectura-, mientras que la función del locutor es desempeñada tanto por los usuarios con más capacidad verbal y dicción como por aquellos otros que tienen dificultades severas para expresarse -solo un 4,2\% de las personas con DI participantes en el cuestionario admitieron que no dominan el habla-. Los beneficiarios que no saben leer, tienen miedo al micrófono o vocalizan con excesivos problemas acomodan sus capacidades a funciones de otro tipo: labor técnica, producción o logística.

Un acercamiento a la participación de estas personas en talleres de radio nos conduce a esta definición de tareas:

- Presentación del programa: Encargado de animar y presentar un programa o una sección de radio. En este punto, comprobamos la existencia de tres figuras: espacios presentados por el locutor de una emisora -locución profesional-, espacios cuya presentación recae en el tutor o coordinador del grupo o programas animados y presentados por un usuario con discapacidad. Un 41,8\% de los participantes con DI o del desarrollo que contestaron a nuestro cuestionario admitieron que presentan o copresentan espacios radiofónicos.

- Elaboración del guion y la escaleta: Existen talleres -principalmente, los que tienen una mayor estabilidad-que cuidan al detalle esta parte de producción. Lo hacen en el curso de una reunión de los integrantes del proyecto en la semana previa o en los días anteriores a la emisión/grabación del espacio. Los tutores nos confirmaron que la guionización del programa es esencial para que los participantes ganen en seguridad, razón por la que no suele dejarse a la improvisación.

- Colaboración en tertulias y secciones de opinión: Hablamos de usuarios que intervienen en espacios de debate. Aunque la preparación del guion es menos exigente, normalmente los participantes preparan los temas que abordan en la charla.

- Realización de entrevistas: Las entrevistas a personajes famosos y a representantes de colectivos de personas con discapacidad son una constante en estos espacios. Lo habitual es que los invitados estén en el estudio de radio y se produzca la buscada interacción social con el entorno. Además, a los colectivos les interesa que el propio entorno conozca de forma presencial la realidad de la discapacidad. 
- Técnico de sonido: Algunos alumnos de los talleres se implican en esta tarea por considerarla atractiva. Aprenden a manejar la mesa de mezclas y las distintas fuentes de sonido, y conocen los más elementales códigos radiofónicos para dirigirse a los locutores. Estamos ante la labor con menor demanda, pues tan solo es realizada por el $8,9 \%$ de los educandos.

- Redacción de noticias, reportajes y crónicas: El nivel de redacción en estos espacios es muy básico, pero son mayoría los talleres en los que se invita a los usuarios a redactar informaciones breves o cuestionarios para entrevistas. La finalidad es que tomen contacto con la actualidad y con los diversos géneros periodísticos. Así, un 45\% de los participantes en los talleres reconocieron participar activamente en esta parte del proceso.

- Locución: Es la tarea preferida. El 98\% de los usuarios, según el cuestionario realizado por los alumnos de los talleres, ejercen esta función.

- Gestión Web: Con la irrupción de Internet, los colectivos dan cada día más relevancia a los pódcast de los espacios y a la distribución de la información en redes sociales. Locutores de las emisoras, tutores de los grupos y usuarios de los talleres realizan este trabajo de postproducción. Es una labor menor, cierto es, como lo demuestra el hecho de que solo el $13,7 \%$ de los alumnos de talleres asegura haber trabajado en esta faceta.

- Producción: El concierto de entrevistas, el manejo de información sobre los temas o la selección de piezas musicales son cuestiones rutinarias de esta holística área de trabajo. Los datos de la encuesta a los alumnos reflejan que un 54\% participan en la producción de los espacios de radio.

\subsection{Motivos de participación}

Para conocer los objetivos que se persiguen con los talleres de radio realizamos una pregunta abierta en el cuestionario propuesto a los tutores-coordinadores para que respondieran sin limitaciones. Posteriormente, ordenamos las respuestas en siete categorías.

- Visibilizar la discapacidad y trasladar una imagen de normalización a la sociedad.

- Empoderar a las personas con discapacidad: trabajo de la autoestima y desarrollo de habilidades comunicativas y sociales.

- Analizar la realidad con espíritu crítico.

- Prevenir el envejecimiento activo.

- Promoción del ocio y tiempo libre.

- Formación laboral de futuros profesionales de los medios. Aprendizaje del proceso informativo-radiofónico.

- Herramienta de difusión para proporcionar información acerca de los colectivos.

Dos son los fines determinantes de los talleres, según la opinión expresada por los tutores. En primer lugar, visibilizar la discapacidad y lanzar una imagen de normalidad a la sociedad en la búsqueda de una representación lo más aproximada posible a 
la realidad que viven las personas con discapacidad. La participación en la radio es un instrumento activo para hacer desaparecer el estigma que separa al entorno social del sujeto con discapacidad. En segundo término, la mayor parte de los coordinadores creen que la comunicación radiofónica puede ser eficaz para empoderar a alumnos con discapacidad al reforzar la identidad individual y mejorar la autoestima. La motivación personal aumenta cuando el educando del taller asume la idea de pertenencia a la comunidad y, en esa línea, la radio es un espacio de encuentro. Además del trabajo psicológico, casi la totalidad de los colectivos sostienen que los talleres radiofónicos mejoran las habilidades comunicativas y sociales en áreas como la comunicación verbal (vocalización, ritmo, tono, énfasis, pausas), lectoescritura y comprensión oral (redacción de textos, locución), creatividad, improvisación, conceptualización de ideas (discernimiento), empatía, asertividad o escucha activa. Un tercer objetivo era analizar la realidad con espíritu crítico dentro de la idea de educomunicación para conocer e interpretar mejor lo que sucede a nuestro alrededor.

Los objetivos que buscan cumplir los tutores fueron confrontados con los motivos que llevan a los usuarios a participar en los talleres. Así, en el cuestionario a los alumnos con discapacidad ofrecíamos seis posibles respuestas:

- Participo para hablar mejor.

- Participo para mejorar la escritura.

- Participo para que me conozcan mejor.

- Participo para sentirme más feliz.

- Participo porque es un tiempo de ocio y entretenimiento.

- Participo para relacionarme más y mejor.

La motivación principal para participar era la experiencia de felicidad que proporciona la radio. El ítem 'Participo para sentirme más feliz' alcanzó un 8,62 sobre 10. La segunda opción elegida, con un 8,36, fue 'Participo porque es un tiempo de ocio y entretenimiento'. También tuvieron una alta votación los ítems 'Participo para relacionarme más y mejor' $(8,15)$ y 'Participo para que me conozcan mejor' $(8,12)$. El aprendizaje de competencias en lectura y escritura eran motivos secundarios.

\section{Discusión y conclusiones}

Aunque algunos talleres han desaparecido en los últimos tiempos y en otros se han producido modificaciones tanto en la forma de organizarse como en el propio nombre del taller, si comparamos los datos de 2014 con los obtenidos en 2017 constatamos que más del $90 \%$ de los proyectos se mantienen, lo que confirma la estabilidad de los talleres radiofónicos. En el estudio inicial se detectaron 38 proyectos de comunicación radiofónica. Aunque se considera que la cantidad es insuficiente teniendo en consideración las dimensiones de Plena inclusión -más de 500 colectivos en España, si excluimos las aproximadamente 300 entidades de Cataluña- y de ASPACE, se atisban nuevas iniciativas al calor de las TIC y de la radio comunitaria y local. El interés por la participación en la radio resulta evidente si observamos que, en el estudio de 
2017 -con la colaboración de Dincat Cataluña y ASPACE-, el número de iniciativas reflejadas superaba el medio centenar (53) -se recabaron datos de unos 20 talleres que no figuraban en la primera consulta-.

La periodicidad para trabajar competencias y fidelizar al oyente es adecuada ya que la mayor parte de los talleres se emiten semanal o quincenalmente o bien varios días a la semana. Esta periodicidad logra así el efecto recuerdo entre la audiencia y sirve para trabajar con regularidad competencias comunicativas y sociales. La duración (de 30 a 60 minutos) también se ajusta a las necesidades de los usuarios -una duración mayor puede provocar pérdidas de concentración y atención en el usuario y una dispersión ambiental-.

Y con respecto al horario, creemos que la participación en horas matinales daría un mayor protagonismo a los colectivos al tratarse de una franja con mayores índices de audiencia.

Centrándonos en la participación, el trabajo en talleres de radio con un intervalo de entre seis y diez usuarios permite una mayor interacción y atención individual a los participantes. Una media de participación más alta no afectaría a uno de los objetivos buscados por los colectivos: visibilizar la discapacidad, pero podría dificultar la consecución de fines personales: empoderamiento del sujeto (desarrollo de habilidades comunicativas y sociales) y aprendizaje del proceso creativo de la radio.

Los talleres son, en su mayoría, jóvenes -las tres cuartas partes tienen menos de cinco años-. Es evidente que las TIC han generado nuevas experiencias radiofónicas, aunque creemos que debe aprovecharse más la radio online, las redes sociales y el formato de podcasting.

Observando el tipo de emisión, se advierte que nueve de cada diez talleres tienen huella en Internet. Eso corrobora el progreso de la e-radio y del pódcast. Su fácil acceso ha democratizado la aparición de micromedios nacidos en Internet -nativos digitales-, pero sería oportuno compatibilizar la presencia en Internet y en la radio convencional -FM-, toda vez que esta última cuenta con un mayor impacto social y el contacto de los usuarios con los profesionales de los medios conduce a un servicio de mayor calidad y a un proceso de aprendizaje más minucioso.

Precisamente, las estaciones de radio comunitarias acogen a una cuarta parte de los talleres. Este tipo de radios prestan un servicio a la pluralidad al estar abiertas a sensibilidades diversas. Por su parte, el número de talleres en emisoras públicas ha crecido en los últimos tres años hasta casi doblar la suma de iniciativas emitidas en estaciones privadas. A pesar de esta corriente, resulta evidente que una parte muy amplia de las emisoras estatales y autonómicas no cumplen con el mandato o fin social al que insta la Ley General de Comunicación Audiovisual puesto que no ofertan en sus programaciones -o lo hacen muy escasamente- espacios dedicados a grupos en riesgo de exclusión social. Dentro de las emisoras públicas cabe subrayar el papel de las estaciones municipales. Debido a su cercanía y a la libertad horaria, están llamadas a ejercer de altavoces de la diversidad.

$\mathrm{Si}$ indagamos en la tipología de los espacios nos cercioraremos de que los programas se suelen emitir en emisoras comunitarias y municipales -programación más diversificada-, mientras que las secciones se pueden escuchar en programas de tipo 
magacín en las desconexiones locales de cadenas de emisoras nacionales. Pensamos que en la sección, a pesar de las limitaciones temporales, se cuenta con el factor diferencial de que se emiten en emisoras de más audiencia (objetivo: visibilizar la discapacidad). Los programas son de mayor duración y se pueden trabajar más competencias (objetivos: empoderamiento; educomunicación), pero generalmente son proyectados en estaciones de menor impacto.

Al estudiar los requisitos de acceso a los talleres nos dimos cuenta de que las entidades exigían filtros mínimos que respondían más a criterios de actitud (compromiso, implicación, trabajo en equipo) que de aptitud. Entre estos últimos podemos hablar de un nivel mínimo de lectoescritura y expresión oral comprensible. Algunas entidades valoran también que el aspirante a entrar en un taller tenga unas mínimas habilidades sociales: trabajar en equipo o relacionarse.

Sin embargo, para los colectivos la participación debe ser un objetivo cardinal sin tener en consideración las limitaciones personales o sociales, en línea con los postulados de la American Association on Intellectual and Developmental Disabilities (AAIDD, 2011). Queda claro, en este caso, que el concepto de participación está indefectiblemente ligado a un sistema de apoyos y oportunidades: "Los modelos mentales del futuro apuestan por un enfoque socioecológico de la discapacidad, énfasis en la inclusión familiar, laboral y social, en la autodeterminación y vida independiente, en un paradigma de apoyos y en centrarse en mejorar la calidad de vida” (Verdugo, 2011: 19).

La experiencia nos ha servido para comprender que no todos los usuarios pueden ejercer la locución o la redacción, pero sí otras funciones dentro del proceso creativo: producción, postproducción, control técnico o gestión web. El fin último es la participación de la mayoría.

Investigar las temáticas que se dan en los programas o secciones nos conduce a una realidad: la mayoría de las entidades dedican parte del tiempo a dar información endógena (sobre el colectivo). Son instrumentos de comunicación interna. En relación a la información exógena o externa, los educandos de los talleres suelen abordar informaciones locales o nacionales, secciones de denuncia social, además de contenidos de ocio y deporte. Son, como se observa, magacines muy variados en los que hay mezcolanza de información y entretenimiento.

Las principales motivaciones para participar en los talleres, según los usuarios, están asociadas a la experiencia de felicidad y el bienestar emocional. Son argumentaciones genéricas, pero enlazan con una reflexión de fondo: la radio es una fuente de estímulos vivenciales. Esto demuestra que el desempeño en la radio es un aprendizaje gratificante: el 97\% de los usuarios participantes en el cuestionario reconocieron que el taller era un hito importante en sus vidas y la valoración media alcanzada fue de 9,24 sobre 10.

Por su parte, los tutores valoraron dos propósitos capitales:

- Visibilizar la discapacidad y trasladar una imagen de normalización a la sociedad. Se persigue aquí luchar contra los estigmas, trabajar en un cambio cultural que mejore la comprensión de la discapacidad.

- Empoderar a las personas con discapacidad. El término empowerment busca, dice Plena inclusión, que las personas crean más en sí mismas, que sean conscientes 
de sus potencialidades. La radio invita a los alumnos a ser constructores de su discurso y puede mejorar la autoestima personal. Dentro del fin del empoderamiento individual y colectivo, los talleres contribuyen también a desarrollar habilidades comunicativas y sociales.

Otros fines como el análisis de la realidad con espíritu crítico dentro de la idea de educomunicación y la promoción del ocio inclusivo son estímulos que permiten al educando mejorar su visión del entorno y aprovechar el tiempo libre en ocupaciones placenteras.

De acuerdo con la teoría de los usos y gratificaciones (McQuail, Brumler y Brown, 1972), los principales objetivos de los talleres serían:

- Concepto de vigilancia: observar la realidad con espíritu crítico.

- Reforzar la identidad personal: empoderamiento.

- Integración e interacción social: mejora de las relaciones sociales.

- Entretenimiento: la radio es un fin en sí mismo que genera ocio.

Estos objetivos, tanto los apuntados por los usuarios como los esgrimidos por los tutores, refuerzan la finalidad de la participación, una de las cinco dimensiones de la American Association on Intellectual and Developmental Disabilities (AAIDD, 2011) al promover "el acceso de las personas con discapacidad a los nuevos sistemas y tecnologías de la información y las comunicaciones, incluida Internet”, tal como significa el artículo 9.g de la Convención sobre los Derechos de las Personas con Discapacidad.

\section{Referencias bibliográficas}

AAIDD (2011). Discapacidad intelectual. Definición, clasificación y sistemas de apoyo (undécima edición). (Trad. M. Á. Verdugo). Madrid: Alianza.

Bonilla del Río, M., Valor Rodríguez, L. y García-Ruiz, R. (2018). Alfabetización mediática y discapacidad. Análisis documental de literatura científica en Web of Science (WoS) y Scopus. Prisma Social: Revista de Investigación Social, 20, 1-20.

Cortés-Fuentes, J. A. (2016). Radio y discapacidad: el taller de radio como agente inclusivo para el empoderamiento de las personas con discapacidad intelectual o del desarrollo. El caso de FEAPS (tesis doctoral inédita). Murcia: Universidad Católica San Antonio (UCAM).

Cortés-Fuentes, J. A. y Correyero-Ruiz, B. (2017). Radio y empoderamiento social. Usos y gratificaciones del taller de radio para las personas con discapacidad intelectual o del desarrollo. El Profesional de la Información, 26 (1), 55-65.

Fernández-SAnde, M. y Leal-Leal, C. M. (2015). La radio como instrumento de rehabilitación y recuperación en trastorno mental severo y crónico: experiencias en la Comunidad de Madrid. En M. Oliveira y F. Ribeiro (Eds.), Radio, sound and Internet, Proceedings of Net Station International Conference (pp. 99-110). Recuperado de http://revistacomsoc. pt/index.php/cecs_ebooks/article/view/2169/2086.

Jiménez-Lara, A. (2011). El estado actual de la Accesibilidad de las Tecnologías de la Información y la Comunicación (TIC). Madrid: CERMI, Fundación Vodafone España. 


\section{EXPERIENCIAS RADIOFÓNICAS CON PERSONAS CON DISCAPACIDAD INTELECTUAL Y PARÁLISIS CEREBRAL EN ESPAÑA \\ JUAN ANTONIO CORTÉS FUENTES Y BEATRIZ CORREYERO RUIZ}

Kaplún, M. (1998). Una pedagogía de la comunicación. Madrid: Ediciones de La Torre.

Katz, E., Gurevitch, M. y HaAs, H. (1973). On the use of the mass media for important things. American Sociological Review, 38 (2), 164-181.

Levín, E. y Denys Espinoza, M. (2009). Enunciar (se) desde la discapacidad. Argumentos (México, D.F.), 22 (61), 141-156.

McQuail, D., Brumler, J. y Brown J. (1972). The Television Audience: a revised perspective. En D. McQuail (Ed.), Sociology of Mass Communications. Harmondsworth: Penguin Books.

ONU (Organización de Naciones Unidas) (1948). Declaración Universal de los Derechos Humanos. Recuperado de http://www.un.org/es/documents/udhr/.

ONU (Organización de Naciones Unidas) (2006). Convención sobre los Derechos de las Personas con Discapacidad. Recuperado de: http://www.un.org/esa/socdev/enable/documents/tccconvs.pdf.

Schalock, R. (2009). La nueva definición de discapacidad intelectual, apoyos individuales y resultados personales. Siglo Cero, 40 (1), 229, 22-39.

Schalock, R. L., Verdugo, M. Á. y Lee, T. (2016). A systematic approach to an organization's sustainability. Evaluation and Program Planning, 56, 56-63.

VArela, J. (2015). La brecha digital en España. Estudio sobre la desigualdad postergada. Madrid: Comisión ejecutiva confederal de UGT. Secretaría de Participación Sindical e Institucional. Recuperado de: https://goo.gl/kpb5VQ.

Verdugo, M. Á. (2011). Dignidad, igualdad, libertad, inclusión, autodeterminación y calidad de vida. Siglo Cero, 42 (4), 240, 18-23. 


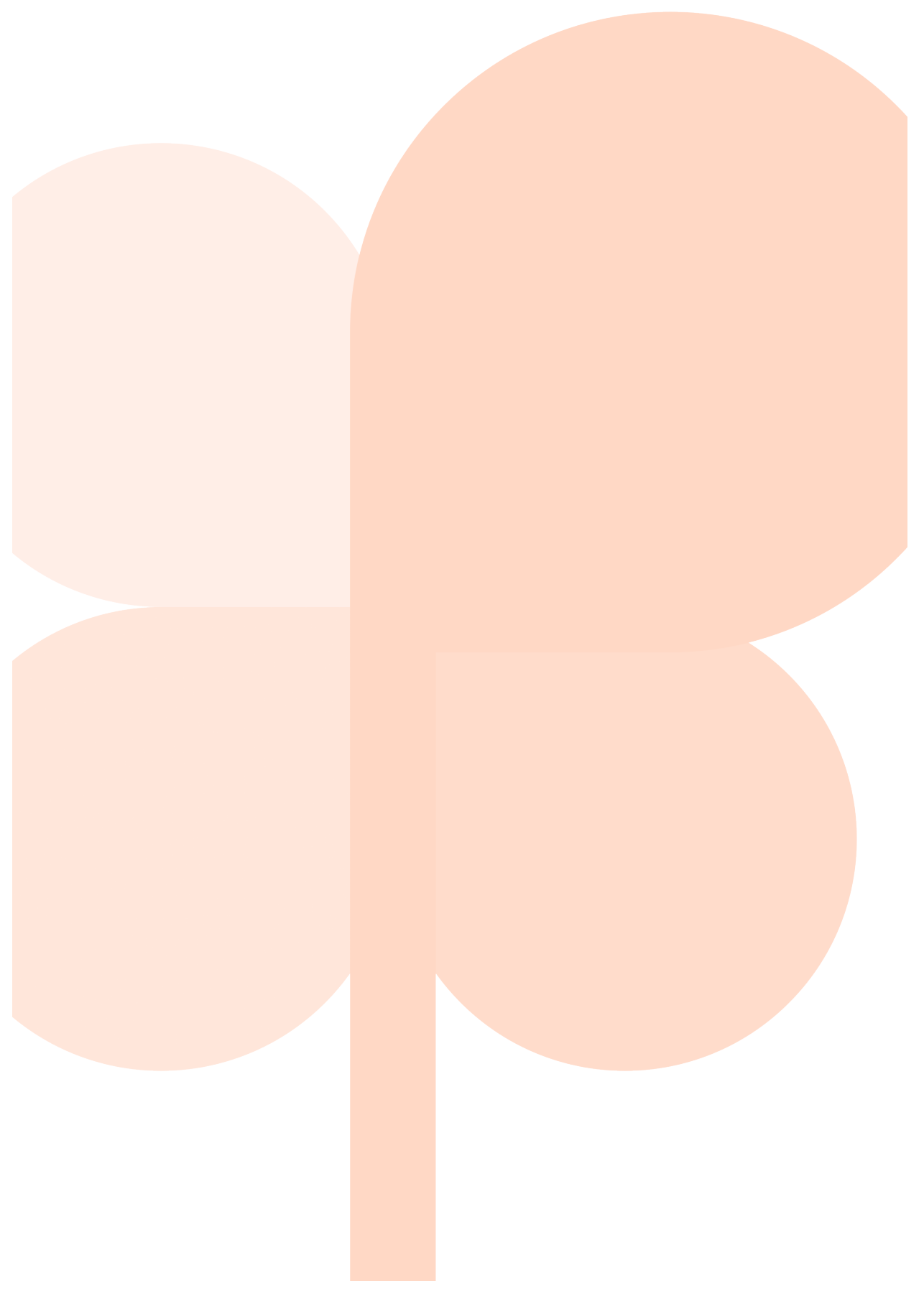

